\title{
Spin-Filtering Effects in Würtzite and Graphite-Like AlN Nanowires with Mn Impurities
}

\author{
G. A. Nemnes \\ Faculty of Physics, "Materials and Devices for Electronics and Optoelectronics" Research Center, University of Bucharest, \\ P.O. Box MG-11, Ilfov 077125 Magurele, Romania
}

Correspondence should be addressed to G. A. Nemnes; nemnes@solid.fizica.unibuc.ro

Received 14 December 2012; Accepted 28 February 2013

Academic Editor: Yun Zhao

Copyright ( 2013 G. A. Nemnes. This is an open access article distributed under the Creative Commons Attribution License, which permits unrestricted use, distribution, and reproduction in any medium, provided the original work is properly cited.

Spin transport properties of magnetic nanowire systems-atomic-sized AlN nanowires with additional Mn impurities-are investigated employing $a b$ initio constrained spin density functional theory calculations and nonequilibrium Green's functions formalism. The analyzed nanowire structures exhibit a stress-induced phase transition, between würtzite and graphite-like configurations. In these quasi-one dimensional systems, the surface states ensure the basic prerequisite in establishing spin and charge transfer, by reducing the relatively large bandgap of the group III nitride semiconductor. The results show in how far this phase transition affects the surface states, focusing on the consequences which appear in the spin-filtering processes.

\section{Introduction}

Group-III nitride nanowires provide nowadays a vast number of applications, ranging from chemical $[1,2]$ and temperature sensors to nanomechanical resonators, thermoelectrical [3] and field emitter [4] applications. In particular, since AlN is a wide bandgap $(\sim 6.2 \mathrm{eV})$ semiconductor, it has become a strong candidate for optoelectronic devices in the UV regime. AlN nanowires are currently produced by a number of techniques, such as self-patterning [5], direct arc discharge [6], or from Al powder [7]. Moreover, inserting transitional metal (TM) impurities (e.g., Mn), attempts have been made in order to produce ferromagnetic quasi-one dimensional diluted magnetic semiconductors [8].

Although the native structure of bulk AlN is würtzite (WZ, space group $\mathrm{Pb}_{3} \mathrm{mc}$ ), it was established that ultrathin nanowires occur naturally in a graphite-like (GL, space group $\mathrm{PG}_{3} / \mathrm{mmc}$ ) phase [9-11]. However, upon applying pulling stress, the structures may retain the WZ configuration. Switching between these two configurations can occur due to thermal fluctuations or mechanically induced stress and this has a large impact in both charge and thermal transport.

The surface states play a significant role in the conduction properties of the nanowires, as they reduce the wide bandgap, which is found in the bulk semiconductor. In contrast to bulk AlN, which has rather good insulating properties, the atomic sized nanowires can achieve reasonable conduction. Therefore, the structures can be regarded as viable solutions for the active region in field effect applications. It is also important to emphasize that it is essential for the nanowire systems to retain a small bang gap (up to $\sim 1-2 \mathrm{eV}$ ), that is, semiconducting behavior, in order to have an efficient field effect control exerted by an external gate.

The AlN nanowires may also provide the low dimensional building blocks for the future spintronic devices, since ferromagnetic ordering has been observed in the bulk semiconductor, doped with Mn impurities [12]. Specific studies on nanowire systems report experimental observation of ferromagnetic ordering [8], as well as the influence of the wire passivation on the stability of the ferromagnetic ground state using $a b$ initio investigations [13]. Nonmagnetic dopants, for example, $\mathrm{Mg}$, can produce unconventional magnetism in AlN nanowire systems, due to the interplay between the low dimensional effects generated by confinement and surface states [14].

In this paper the spin filtering effects are investigated in AlN atomic-sized nanowires with additional Mn impurities, with the main focus on the differences which arise by switching between the two structural configurations, WZ and GL. The GL phase corresponds to the freestanding nanowire, 


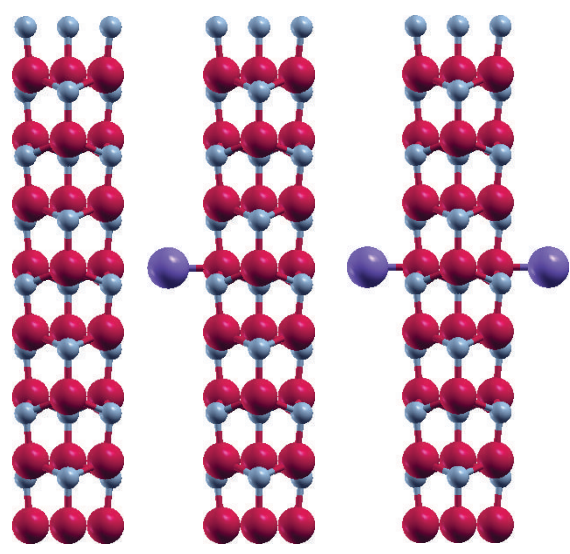

FIGURE 1: Pristine WZ nanowire and structures with one or two Mn impurities.

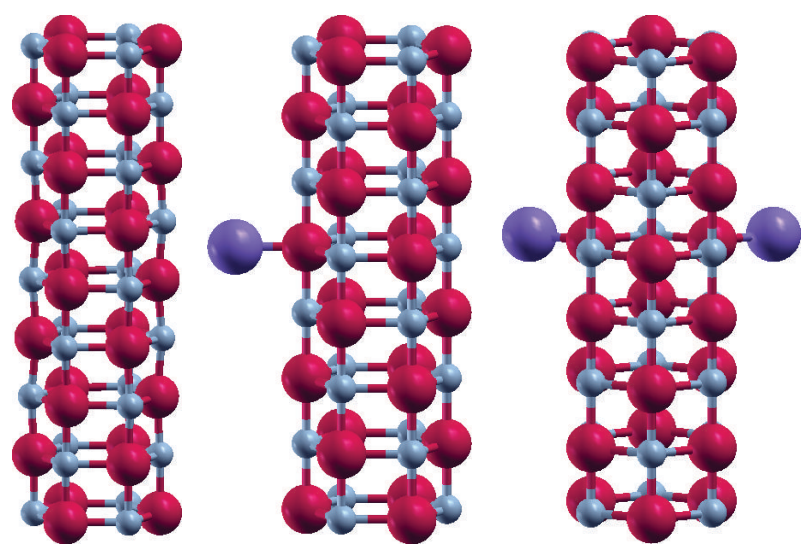

Figure 2: Pristine GL nanowire and structures with one or two Mn impurities.

while the WZ phase is obtained by tuning the applied axial stress. The contribution of the surface states to the spin transport is comparatively analyzed. The results convey the possibility of using AlN nanowires as nanomechanical spintronic devices, where the spin detection can indicate the magnetic and structural state of the system.

\section{Structures and Methods}

The structures considered are AlN nanowires with one or two additional impurities, depicted in Figures 1 and 2. The GL configurations correspond to free-standing wires, while the WZ structures are obtained by applying an external pulling stress. The nanowires are functionalized by introducing additional Mn impurities on the surface. Depending on the magnetic moments of the impurities the two components of spin current are scattered differently. For the systems considered here it is expected that the transmission is less affected compared to structures with substitutional TM impurities. However, one needs to asses in how far the adatoms can still produce a reasonable spin current polarization and, furthermore, evaluate the potential differences introduced by switching between the two structural configurations.
The $a b$ initio calculations are performed in the framework of density functional theory (DFT), using the SIESTA package [15]. The magnetic structures are investigated using constrained spin-DFT calculations, in the local spin density approximation (LSDA), with the parametrization proposed by Ceperley and Alder. In the supercell approach implemented by SIESTA, the real space grid was set by a cutoff energy of $300 \mathrm{Ry}$, and a Monkhorst-Pack scheme to $1 \times$ $1 \times 5 k$-points was considered. In order to obtain the atomic coordinates, structural relaxations are performed. The starting point is the pristine AlN nanowire in the WZ phase, which is the native configuration of the bulk material. The initial lattice parameters correspond to the bulk system, that is, $a_{0}^{\mathrm{WZ}}=3.112 \AA, c_{0}^{\mathrm{WZ}}=4.982 \AA$. The system is relaxed using a maximum displacement of $0.1 \AA$ until the forces are less than $0.04 \mathrm{eV} / \AA$. The final configuration obtained for the freestanding wire is the hexagonal GL phase. In the GL structure the $\mathrm{Al}$ and $\mathrm{N}$ atoms are placed in alternating positions in hexagonal layers, with the lattice constant $a^{\mathrm{GL}}=1.97 \AA$, that is, the in-plane distance between two successive $\mathrm{Al}$ and $\mathrm{N}$ atoms. The distance between the hexagonal layers, which are equally spaced, is $c^{\mathrm{GL}}=1.87 \AA$. The lattice constant along the wire direction is therefore reduced from $4.982 \AA$ to $2 c^{\mathrm{GL}}=3.74 \AA$. However, by applying a pulling force of $0.40 \mathrm{nN}$, the wire retains the original $\mathrm{WZ}$ configuration, with slightly modified lattice parameters, $a^{\mathrm{WZ}}=2.79 \AA$, $c^{\mathrm{WZ}}=4.86 \AA$. The lattice parameters generally depend on the applied axial stress.

The spin dependent transmission functions are extracted using the non-equilibrium Green's functions (NEGF) formalism, which is implemented in TRANSIESTA [16]. The scattering region consists of four unit cells, with a total of 48 atoms and it is coupled to the left/right semi-infinite electrodes. The effects introduced by the additional Mn impurities, which are present on the nanowire surface, can be observed in the calculated transmission functions corresponding to the two spin channels, $T_{\uparrow}$ and $T_{\downarrow}$. The total transmission function can be found for a total energy $E$ using the relation

$$
T(E)=\operatorname{Tr}\left[\Gamma_{L} G^{r} \Gamma_{R} G^{a}\right],
$$

where $G^{r / a}$ are the retarded/advanced Green's functions and $\Gamma_{L / R}$ are the self-energies corresponding to the couplings with the two contacts. In linear regime, the efficiency of a spinfilter device can be defined using the transmission of each spin component [17]:

$$
p^{s}=\frac{T_{\uparrow}-T_{\downarrow}}{T_{\uparrow}+T_{\downarrow}},
$$

where $s$ indicates the structural configuration, $s=$ WZ, GL.

\section{Results and Discussion}

As it was already pointed out in the introduction, the surface states which appear in the considered quasi-one-dimensional structures effectively reduce the bandgap of the aluminium nitride. Figure 3 shows the total and partial densities of 


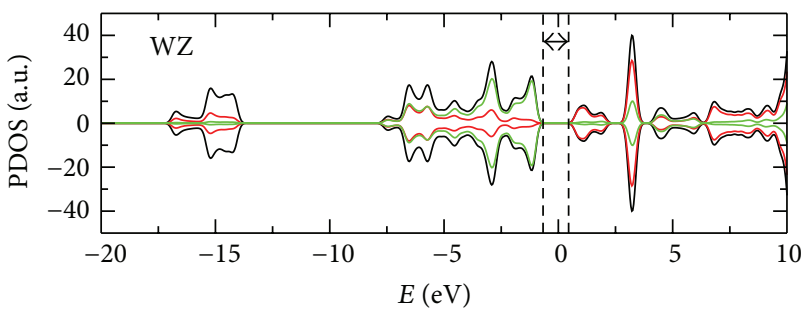

(a)

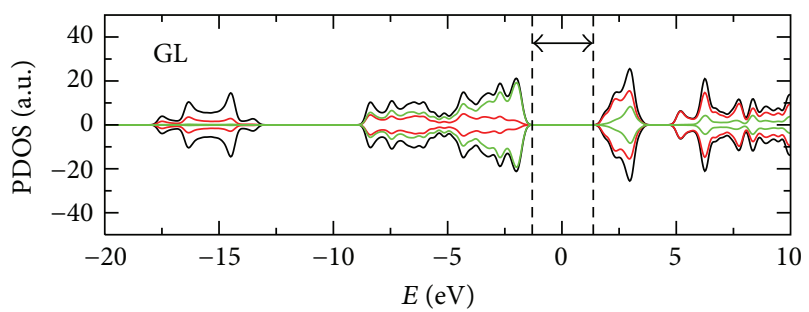

(b)

FIGURE 3: Total (black) and partial density of states for WZ and GL structural configurations. The contribution of the $p$ orbitals of nitrogen (green) and $s, p$ orbitals of aluminium (red) are represented. The arrows mark the minibandgaps between the valence band and the first group of surface states.
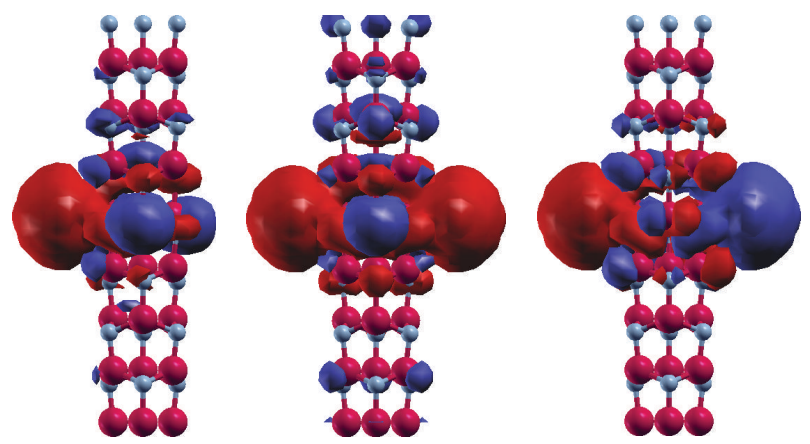

FIgURE 4: Spin densities for nanowires in the WZ configuration, with one and two Mn impurities. Both ferro- and antiferromagnetic configurations are indicated.

states (DOS) of the ideal nanowires, for the two structural configurations. One can see that the mini-bandgaps which appear, $E_{g}^{\mathrm{WZ}} \approx 1.2 \mathrm{eV}$ and $E_{g}^{G L} \approx 2.5 \mathrm{eV}$, are significantly smaller than the bandgap of bulk AlN. In both WZ and GL phases, the valence band is formed by $p$ orbitals of nitrogen. For the WZ nanowire the first group of surface states is formed by both $s$ and $p$ orbitals of aluminium, while for the GL configuration there is also a noticeable contribution from the $p$ orbitals of nitrogen. It is important to note that in the case of WZ nanowire there is an effective bandgap comparable with the one of bulk silicon, which suggests the atomic sized structure is suitable for spin and charge transfer applications.

Next, by introducing the Mn adatoms, the spin densities are comparatively analyzed for WZ and GL structures and a clear qualitative difference can be observed in Figures 4 and

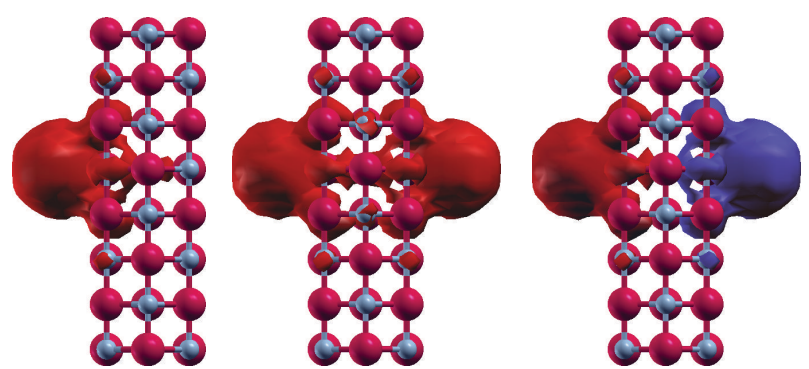

FIGURE 5: Spin densities for nanowires in the GL configuration, with one and two Mn impurities. Both ferro- and antiferro-magnetic configurations are indicated.

5. For the two impurity cases, both ferro- (FM) and antiferromagnetic (AFM) configurations of the magnetic moments are investigated. As expected, the spin polarization is maximum at the location of the magnetic impurities. In the case of WZ nanowire, there is also a large polarization of neighboring $\mathrm{Al}$ and $\mathrm{N}$ atoms, which are otherwise nonmagnetic. Opposite spin polarizations can be observed at the first and second order neighbors. This is in contrast with the case of the GL structure, which has better insulating properties and the net spin density remains confined at the magnetic adatoms. In the case of two impurities, placed symmetrically to one of the planes which bisect the structures in two identical halves, a mirrored symmetry in the spin density can be noticed. This has consequences in the spin transport, where both components of spin current are equally transmitted, that is, the net spin current is zero. Thermal excitations as well as small magnetic fields can switch the magnetic state of the impurities from FM to AFM, turning on and off the spin current. This suggests the possibility of using the considered magnetic nanowires as building blocks for high sensitivity temperature and magnetic field sensors.

The spin resolved transmission is depicted in Figures 6 and 7 , for systems with one and two magnetic impurities, respectively. The pristine non-magnetic nanowires are first analyzed in both WZ and GL configurations. In this case each propagating mode adds one unit plateau to the total transmission. The ideal transmission follows closely the density of states plotted in Figure 3. The adatoms placed on the nanowire surface introduce a selective scattering on the two spin components, depending on the preset magnetic configuration. Contrary to the situation where the magnetic impurities are placed substitutional, in this case the transmission is rather close to ideal. More significant scattering is visible in the energy range corresponding to the first group of surface states. The mini-gaps are consistent with the previously obtained values from the DOS analysis. For the systems with the pair of magnetic impurities in the AFM configuration, there is a rather similar transmission function with the one indicated in Figures 6 and 7, with major qualitative difference that in this case one has $T_{\uparrow}=T_{\downarrow}$.

The spin polarization depends on the intricate scattering processes, which follow from the addition of magnetic impurities and which are also dependent on the magnetic configuration. Due to the sharp variations present in the transmission 


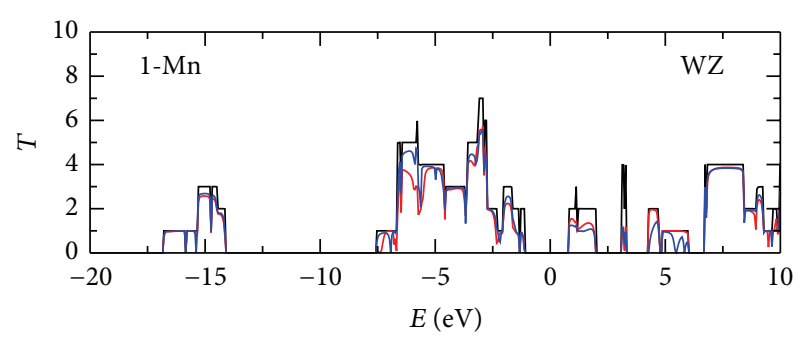

(a)

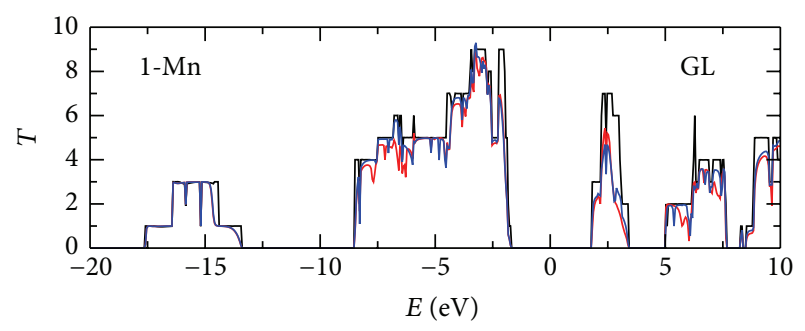

(b)

FIGURE 6: Transmission versus energy for nanowires in the WZ and GL configurations: pristine nanowire (black) and nanowires with one $\mathrm{Mn}$ impurity; $T_{\uparrow}$ (red) and $T_{\downarrow}$ (blue) are represented. The Fermi level is located at $E=0$.

functions, polarizations close to unity are possible. However, they occur on small energy ranges. Therefore the data indicated in Figure 8 was averaged on energy intervals of $~$ $25 \mathrm{meV}$, consistent with realistic thermal fluctuations. By this procedure, spin polarizations above $20 \%$ can still be achieved for the GL structures. Focusing on the first group of surface states, one can notice that the differences between the oneand two-impurity cases are smaller for the GL structure, when compared to its WZ counterpart. This indicates the potential robustness of the spin characteristics with respect to adding one extra impurity.

To conclude, both WZ and GL structures with Mn adatoms exhibit reasonable transmission and are able to produce detectable spin polarization. The reduced bandgaps offer the possibility of using the magnetic AlN nanowires in spintronic field effect applications.

\section{Conclusions}

AlN nanowires with Mn adatoms are investigated using constrained spin-DFT calculations. The phase transition between the würtzite and graphite-like configurations is discussed in the context of exploring the spin transport properties. A general feature of the analyzed quasi-one dimensional structures is the reduction of the band gap, down to $\sim 1.2 \mathrm{eV}$ for the WZ nanowire. Consequently, although bulk AlN is a wide bandgap semiconductor, it is suitable for atomic sized devices which employ the field effect. With the additional impurities placed on the surface of the nanowires, the scattering is reduced and the transmission is at a significant ratio, when compared with the ideal case. However, one or two Mn impurities in the ferromagnetic configuration still provide a reasonable spin separation and the net current may be further

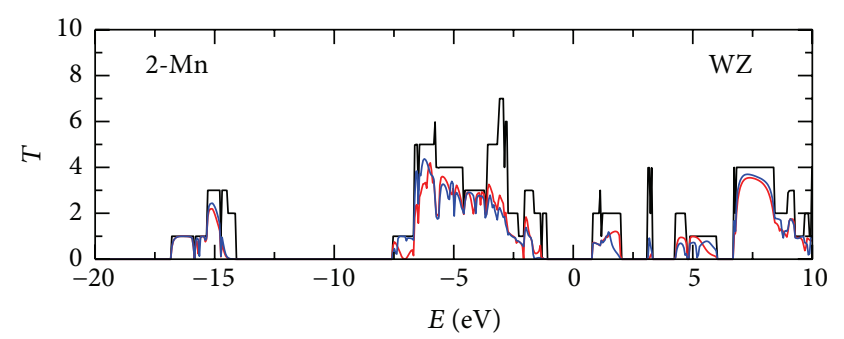

(a)

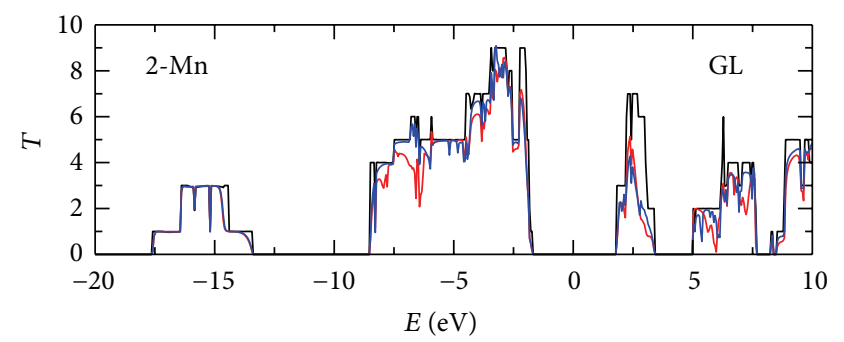

(b)

FIGURE 7: Similar data as in Figure 6 for nanowires with two Mn impurities, in the ferromagnetic configuration.

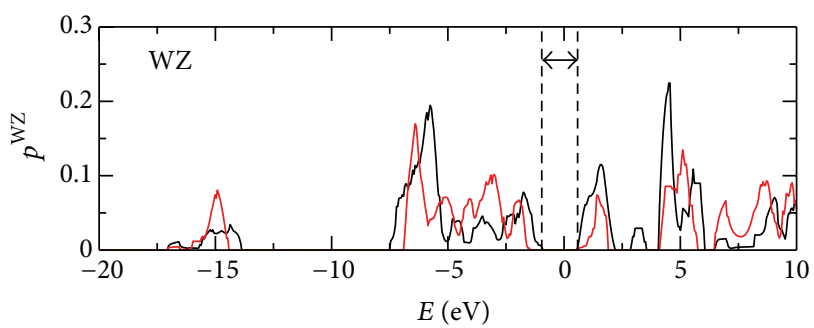

(a)

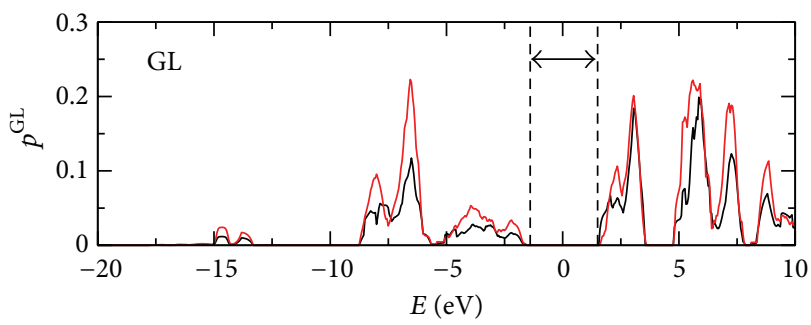

(b)

FIGURE 8: Spin polarization (absolute value) for WZ and GL nanowires with one (black) or two (red) impurities. The arrows indicate the mini-bandgaps.

manipulated in a spintronic device. Taking advantage of the symmetries, it was shown that the considered structures in the antiferro magnetic configurations provide zero net spin current. By switching the magnetic states of the impurities, a detection mechanism is provided. Applying axial stress, the changes in the spin transfer characteristics can be pointed out. The analyzed nanowire systems can therefore provide the building blocks for integrating both nanomechanical and spintronic devices. 


\section{Acknowledgments}

This work was supported by the Romanian National Authority for Scientific Research, CNCS-UEFISCDI, Projects nos. PN-II-RU-PD-2011-3-0044 and PN-II-ID-PCE-2011-3-0960 and in part by the European Commission under EU FP7 Project HP-SEE (under Contract no. 261499).

\section{References}

[1] S. Chattopadhyay, C. S. Shih, H. L. Zon, F. C. Chia, K. H. Chen, and L. C. Chen, "Molecular sensing with ultrafine silver crystals on hexagonal aluminum nitride nanorod templates," Journal of the American Chemical Society, vol. 127, no. 9, pp. 2820-2821, 2005.

[2] Z. Zhou, J. Zhao, Y. Chen, P. von Ragu Schleyer, and Z. Chen, "Energetics and electronic structures of AlN nanotubes/wires and their potential application as ammonia sensors," Nanotechnology, vol. 18, Article ID 424023, 2007.

[3] G. A. Nemnes, C. Visan, and S. Antohe, "Thermopower of atomicsized wurtzite AlN wires," Physica E, vol. 44, p. 1092, 2012.

[4] C. Xu, L. Xue, C. Yin, and G. Wang, "Formation and photoluminescence properties of AlN nanowires," Physica Status Solidi (A), vol. 198, no. 2, pp. 329-335, 2003.

[5] G. R. Yazdi, M. Syvajarvi, and R. Yakimova, "Aligned AlN nanowires and microrods by self-patterning," Applied Physics Letters, vol. 90, Article ID 123103, 2007.

[6] L. H. Shen, X. F. Li, J. Zhang et al., "Synthesis of singlecrystalline wurtzite aluminum nitride nanowires by direct arc discharge," Applied Physics A, vol. 84, pp. 73-75, 2006.

[7] R. K. Paul, K. H. Lee, B. T. Lee, and H. Y. Song, "Formation of AlN nanowires using Al powder," Materials Chemistry and Physics, vol. 112, no. 2, pp. 562-565, 2008.

[8] Y. Yang, Q. Zhao, X. Z. Zhang et al., "Mn-doped AlN nanowires with room temperatureferromagnetic ordering," Applied Physics Letters, vol. 90, Article ID 092118, 2007.

[9] L. H. Shen, X. F. Li, Y. M. Ma et al., "Pressure-induced structural transition in AlN nanowires," Applied Physics Letters, vol. 89, Article ID 141903, 2006.

[10] Y. Wu, G. Chen, H. Ye, Y. Zhu, and S. H. Wei, "Origin of the phase transition of AlN, GaN, and $\mathrm{ZnO}$ nanowires," Applied Physics Letters, vol. 94, Article ID 253101, 2009.

[11] T. L. Mitran, A. Nicolaev, G. A. Nemnes, L. Ion, and S. Antohe, "Ab initio vibrational and thermal properties of AlN nanowires under axial stress," Computational Materials Science, vol. 50, no. 10, pp. 2955-2959, 2011.

[12] H. Li, H. Q. Bao, B. Song et al., "Ferromagnetic properties ofMndoped AlN," Physica B, vol. 403, pp. 4096-4099, 2008.

[13] Y. Zhang, H. Shi, R. Li, and P. Zhang, "Magnetic coupling properties of Mn-doped AlN nanowires: first-principles calculations," Physics Letters A, vol. 375, no. 15, pp. 1686-1689, 2011.

[14] Z.-K. Tang, L. Wang, L.-M. Tang et al., "Ferromagnetic coupling in Mg-doped passivated AlN nanowires: a firstprinciples study," Physica Status Solidi B, vol. 249, pp. 185-189, 2012.

[15] J. M. Soler, E. Artacho, J. D. Gale et al., "The SIESTA method for ab initio order-N materials simulation," Journal of Physics Condensed Matter, vol. 14, no. 11, pp. 2745-2779, 2002.

[16] M. Brandbyge, J. L. Mozos, P. Ordejon, J. Taylor, and K. Stokbro, "Density-functional method for nonequilibrium electron transport," Physical Review B, vol. 65, Article ID 165401, 17 pages, 2002.
[17] G. A. Nemnes, "Spin current switching and spin-filtering effects in Mn-doped boron nitride nanoribbons," Journal of Nanomaterials, vol. 2012, Article ID 748639, 5 pages, 2012. 

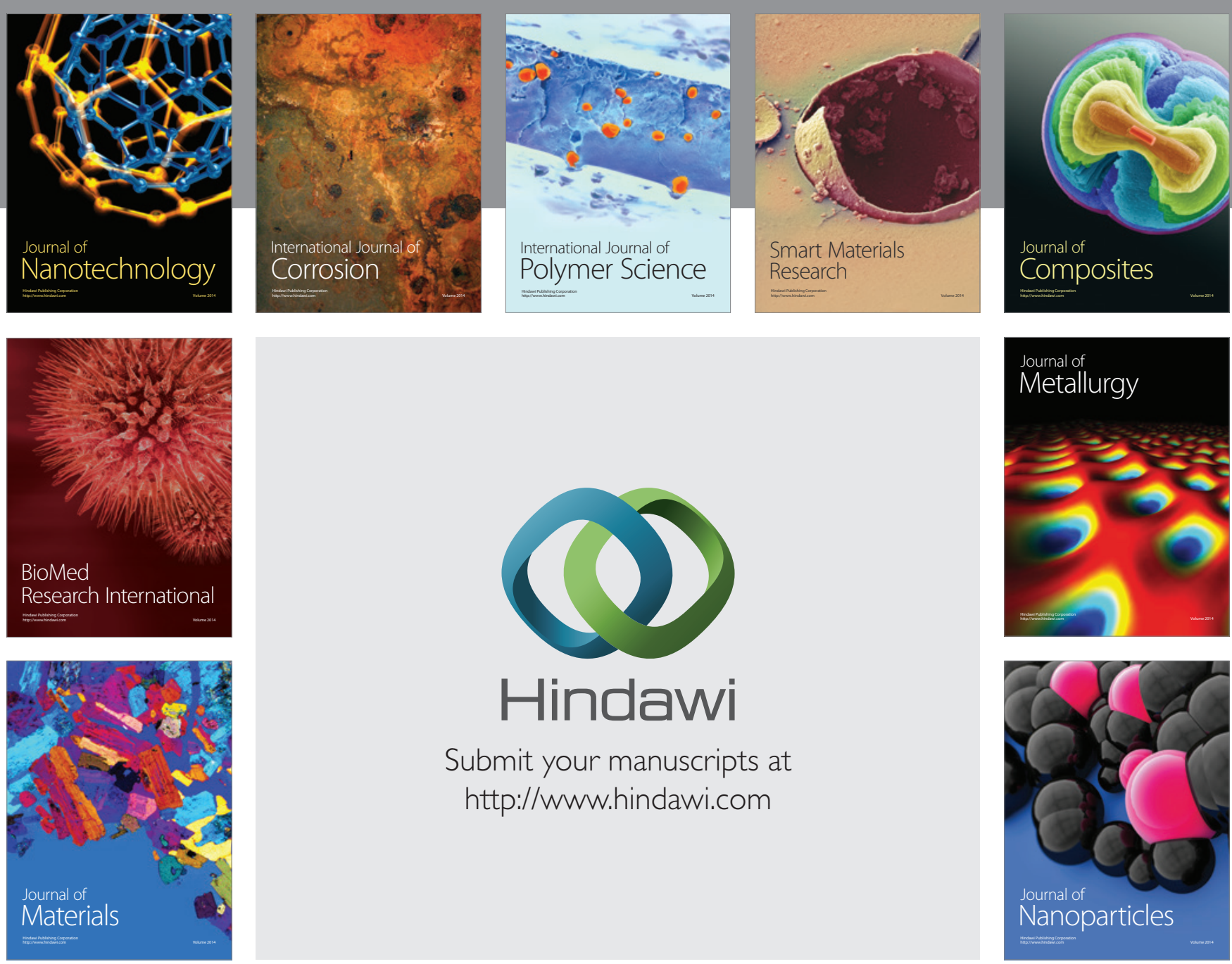

Submit your manuscripts at http://www.hindawi.com
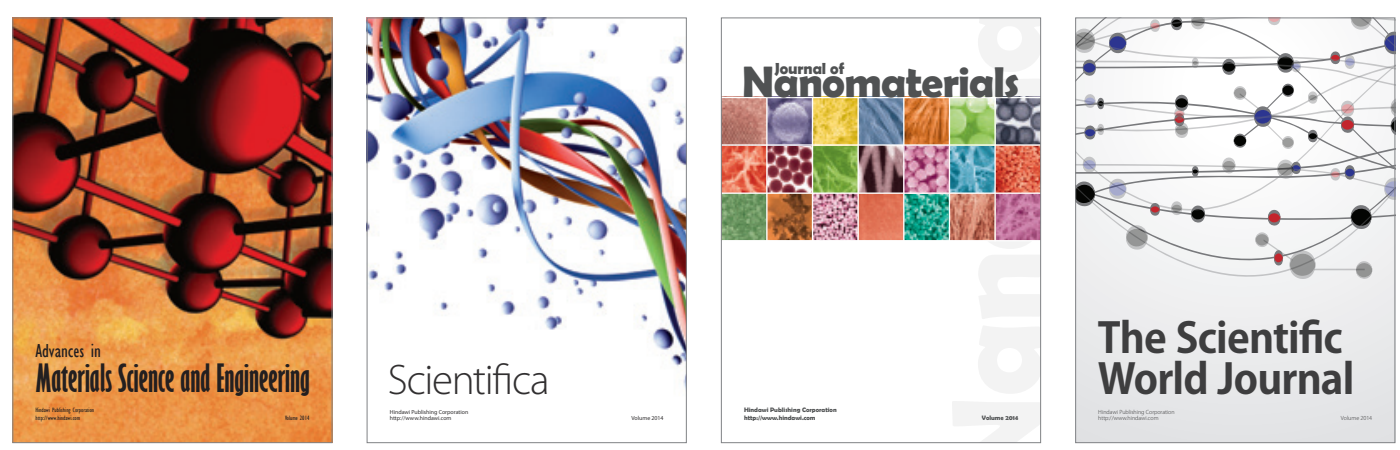

\section{The Scientific World Journal}
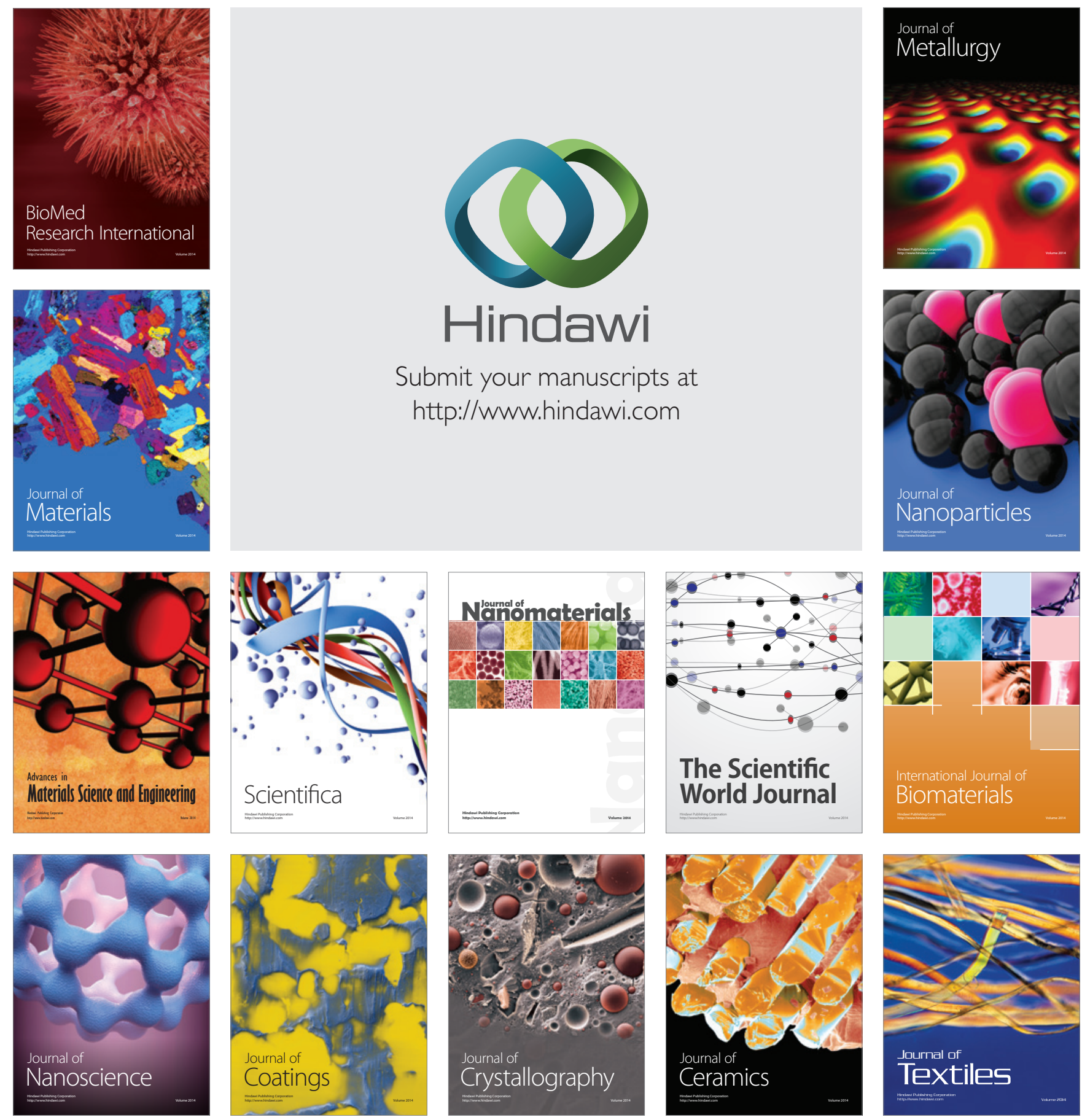